\section{Livraisons}

d'Histoire

de l'Architecture

\section{Livraisons de l'histoire de l'architecture}

19 | 2010

Du remploi à l'éphémère

\title{
Un joyau éphémère de l'Art nouveau : le pavillon bleu à l'Exposition universelle de 1900
}

A fleeting jewel of Art nouveau: the Pavillon bleu at the Universal Exposition of 1900

Ein vergängliches Kleinod des Jugendstils : der Pavillon bleu der Weltausstellung 1900

Marie-Amélie Tharaud

\section{OpenEdition}

\section{Journals}

Édition électronique

URL : http://journals.openedition.org/lha/246

DOI : $10.4000 /$ lha. 246

ISSN : 1960-5994

Éditeur

Association Livraisons d'histoire de l'architecture - LHA

Édition imprimée

Date de publication : 10 juin 2010

Pagination : 135-147

ISSN : 1627-4970

Référence électronique

Marie-Amélie Tharaud, « Un joyau éphémère de l'Art nouveau : le pavillon bleu à l'Exposition universelle de 1900 », Livraisons de l'histoire de l'architecture [En ligne], 19 | 2010, mis en ligne le 10 juin 2012 , consulté le 30 avril 2019. URL : http://journals.openedition.org//ha/246 ; DOI : 10.4000//ha.246

Ce document a été généré automatiquement le 30 avril 2019

Tous droits réservés à l'Association LHA 


\title{
Un joyau éphémère de l'Art nouveau : le pavillon bleu à l'Exposition universelle de 1900
}

\author{
A fleeting jewel of Art nouveau: the Pavillon bleu at the Universal Exposition of \\ 1900
}

Ein vergängliches Kleinod des Jugendstils : der Pavillon bleu der Weltausstellung 1900

Marie-Amélie Tharaud

\begin{abstract}
À côté des palais officiels français et des pavillons nationaux, le Pavillon bleu (ill. 1) se démarque de l'éclectisme dominant à l'Exposition universelle de 1900 pour afficher un style Art nouveau éclatant. Bâti près d'un lac artificiel au pied de la tour Eiffel, ouvert le jour de l'inauguration de l'Exposition, le 14 avril 1900, la vocation du Pavillon bleu n'en est pas moins futile et mercantile: c'est un restaurant de luxe, véritable enseigne publicitaire pour un restaurant du même nom établi à Saint-Cloud. Première œuvre conjointe du décorateur belge Gustave Serrurier-Bovy et de l'architecte français René Dulong, elle est l'une des créations architecturales les plus audacieuses de l'Exposition, la démonstration virtuose d'un Art nouveau de grande qualité.
\end{abstract}


III. 1 : Pavillon bleu de l'Exposition universelle de 1900, architecte René Dulong, décorateur Gustave Serrurier-Bovy

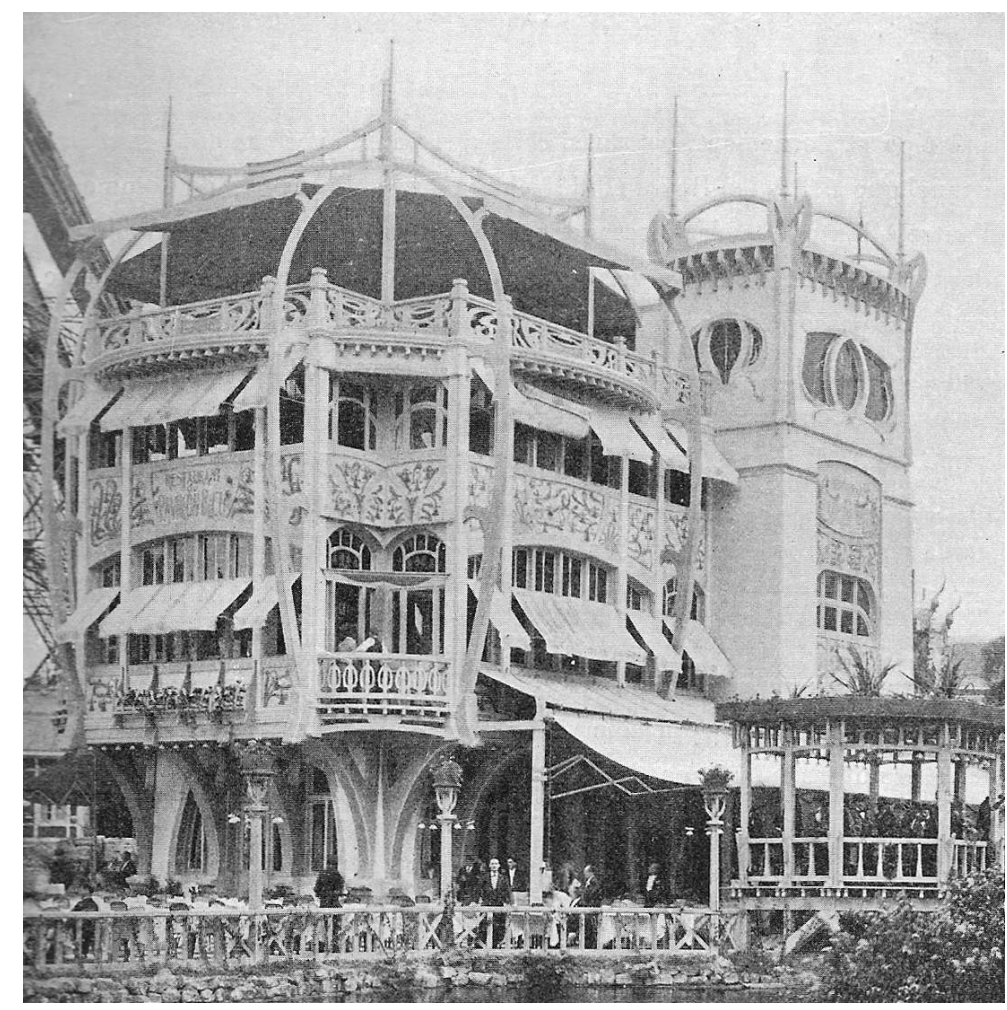

DANS ROgER MARX, LA DÉCORATION ET LES INDUSTRIES D'ART À L'EXPOSITION UNIVERSELLE DE 1900, PARIS, CH. DELAgRAVE, 1901, P. 27.

Pourtant, exceptés les ouvrages de Françoise Bigot du Mesnil du Buisson et d'Étienne du Mesnil du Buisson consacrés à Gustave Serrurier ${ }^{1}$, auxquels cet article est redevable, le Pavillon bleu demeure un édifice méconnu de l'histoire de l'Art nouveau, car sa disparition précoce et peu renseignée a contribué à son oubli : l'édifice, comme tous ceux de l'Exposition - hormis le Grand Palais, le Petit Palais et le pont Alexandre III - fut rapidement détruit. Aussi les photographies d'époque en noir en blanc sont-elles insuffisantes à rendre l'attrait d'une architecture qui faisait la part belle à la polychromie.

En outre, non seulement la vocation ludique et la visibilité du Pavillon bleu en font l'un des édifices Art nouveau les plus remarqués de l'Exposition universelle, mais il constitue également la principale démonstration d'un Art nouveau franco-belge dans cette manifestation où les rivalités nationalistes sont cruciales.

\section{De Saint-Cloud à l'Exposition universelle}

4 Le Pavillon bleu est d'abord une entreprise publicitaire. Son concessionnaire, Charles Moreux, possède à l'entrée du parc de Saint-Cloud un restaurant du même nom, alors célèbre : un guide évoque ainsi «l'établissement si connu de Saint-Cloud» et prédit au Pavillon bleu de l'Exposition "le même succès que son aîné ${ }^{2}$. Ce dernier est un édifice cossu, doté d'une large coupole d'angle, de balustrades ajourées et de grandes fenêtres. Datable des années 1880 ou 1890, son style n'a aucun lien avec l'Art nouveau. Le Pavillon bleu de l'Exposition universelle ne cherche donc pas à rappeler l'allure de celui de Saint- 
Cloud, mais affirme, par son style, la modernité de l'établissement pour attirer une clientèle bourgeoise. En effet, parmi les trois catégories de restaurants définies par le commissariat général, établissements de luxe, à prix moyens, ou populaires ${ }^{4}$, le Pavillon bleu de l'Exposition se situe dans la première, ce que confirment divers guides :

[...] un pavillon original où les visiteurs de l'Exposition trouveront un service confortable et luxueux fait avec un matériel d'un style "art nouveau » des plus gracieux et aux sons d'un entraînant orchestre de tziganes ${ }^{5}$.

5 Par ailleurs, deux menus conservés aux Archives de Paris montrent bien le luxe de la carte - même s'il s'agit là plutôt de réceptions particulières ${ }^{6}$. Alors qu'on ignore comment Charles Moreux est entré en contact avec René Dulong et Gustave Serrurier, le luxe du Pavillon bleu s'affirme aussi dans une architecture et une décoration particulièrement soignées en dépit de leur caractère éphémère.

\section{RENÉ DULONg ET GUSTAVE SERRURIER}

\section{RÉPARTITION DES RÔLES}

6 L'adjudication des restaurants français a lieu le 19 août $1899^{7}$. Le 22 septembre, Moreux obtient que la surface de son emplacement passe de 100 à $152 \mathrm{~m}^{28}$. La construction du Pavillon bleu n'a sûrement pas débuté avant cette date, et le restaurant a ouvert le 14 avril $1900^{\circ}$ : les travaux ont donc duré moins de sept mois, preuve qu'ils ont été menés avec efficacité et que la collaboration entre Dulong et Serrurier a bien fonctionné. Seul le critique A. Barthélémy, qui paraît être une source fiable ${ }^{10}$, décrit avec précision la répartition des rôles entre eux :

Pour la décoration peinte, morceaux de frise courant à l'intérieur ou à l'extérieur, comme pour l'ameublement et les appareils électriques, M. René Dulong a joui de la collaboration de M. Serrurier ${ }^{11}$.

7 D'autres documents identifient Dulong comme l'architecte, la collaboration de Serrurier n'étant pas toujours mentionnée ${ }^{12}$. Cette répartition, Dulong architecte et Serrurier décorateur, n'exclut bien sûr pas une réflexion commune : les lignes de l'édifice évoquent les meubles de Serrurier et, dans une moindre mesure, les arches de la salle des importations conçue par Serrurier pour l'exposition de Tervueren. À l'inverse, Dulong n'a pas eu de lien avec l'Art nouveau auparavant. Roger Marx, grand défenseur de l'Art nouveau, estime du reste que «bien que M. Serrurier n'intervienne que comme collaborateur de M. Dulong, et pour la partie ornementale, l'ensemble paraît un compromis entre l'art de Belgique et celui de Paris ${ }^{13}$ ». Un travail en commun est d'autant plus plausible que les deux hommes se sont associés dans une galerie, "L'Art dans l'habitation », en avril 1900.

\section{HISTOIRE D'UNE ASSOCIATION}

8 Il semble que Dulong et Serrurier se soient rencontrés en 1899. Ils ont alors suivi des parcours très différents. Après des études à l'École des beaux-arts, René Dulong (1860-1944) mène une carrière traditionnelle, et peu éclatante, au sein du service d'architecture de la Ville de Paris ${ }^{14}$. Il se spécialise dans l'architecture hospitalière et sociale (asiles, stations d'ambulances, crèche, orphelinat), exerçant souvent des missions d'inspection. À l'Exposition universelle de 1900, il occupe un poste d'inspecteur dans 
l'agence centrale d'architecture ${ }^{15}$ et, en plus du Pavillon bleu, édifie le Phono-cinéma-

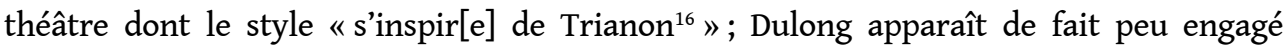
stylistiquement. Il devient à cette époque le concessionnaire de Serrurier pour la galerie "L'Art dans l'habitation", rue de Tocqueville à Paris, puis son associé avec Alphonse Verstraete sous la dénomination "Serrurier et Cie ", de 1903 à 1907. Ses archives ayant disparu ${ }^{17}$, la suite de la vie de Dulong est moins connue : on sait qu'il travaille à Buenos Aires de 1910 à 1913, puis qu'il obtient à nouveau une fonction officielle à l'Exposition des arts décoratifs de $1925^{18}$.

9 Gustave Serrurier-Bovy (1858-1910) a également débuté par l'architecture, pour se tourner ensuite vers les arts décoratifs ${ }^{19}$. Il étudie à l'Académie royale des beaux-arts de Liège puis travaille comme architecte dans cette ville. Mais il abandonne cette carrière en 1888 à la suite d'un conflit administratif autour d'un projet d'hôpital. Il prend dès lors en main le magasin de décoration de sa femme, Maria Bovy, et après quelques séjours en Angleterre, se met à dessiner des meubles inspirés des Arts and Crafts. Il rencontre vite le succès dans les expositions de la Libre Esthétique, et s'oriente vers des productions semisérielles recherchant "l'art pour tous". Sa réputation confortée à l'exposition de Tervueren en 1897, il ouvre une galerie à Bruxelles. Après sa rencontre avec Dulong, Serrurier se tourne davantage vers la France: il décore le château de La Chapelle-enServal en 1901, puis autour de 1905 celui de La Cheyrelle, propriété de la belle-famille de Dulong, ce dernier dirigeant les travaux. Enfin, avec la fin de la société « Serrurier et $C^{\text {ie }}$ ", Serrurier poursuit seul ses activités sous la dénomination "Serrurier-Bovy " jusqu'à sa mort en 1910.

Les circonstances de la rencontre entre Dulong et Serrurier demeurent mystérieuses, tout comme la part respective de l'un et de l'autre dans les créations de leur association. Le Pavillon bleu est en tout cas l'œuvre phare de leur rencontre et révèle toute l'harmonie de leur collaboration.

\section{Le Pavillon bleu}

11 En l'absence de plan détaillée ${ }^{20}$, les photographies et divers témoignages apportent des renseignements assez précis pour l'extérieur, très partiels pour l'intérieur ${ }^{21}$. S'étendant sur $152 \mathrm{~m}^{2}$, le Pavillon bleu prend la forme d'un plan carré flanqué de deux tours d'angles côté ouest. Il comporte deux niveaux de dépendances en sous-sol, un rez-de-chaussée et deux étages de salles de restaurant, ainsi qu'une terrasse sous un velum soutenu par de grandes arches qui parcourent toute la façade. Tandis que le plan carré assure une forme régulière, l'imposant encorbellement de deux mètres sur le rez-de-chaussée vient troubler cette stabilité et donne de l'essor à l'édifice - tout en permettant un gain d'espace pour la même surface au sol. Trois façades identiques, selon une répétition gracieuse, laissent une place prépondérante aux ouvertures. La quatrième façade (ill. 2), comprise entre les deux tours, diffère: composée de petites fenêtres disposées symétriquement, elle correspond aux espaces de passage ${ }^{22}$. Les tours, qui abritent les escaliers ${ }^{23}$, surplombent le velum et apportent élan et majesté à l'ensemble. 


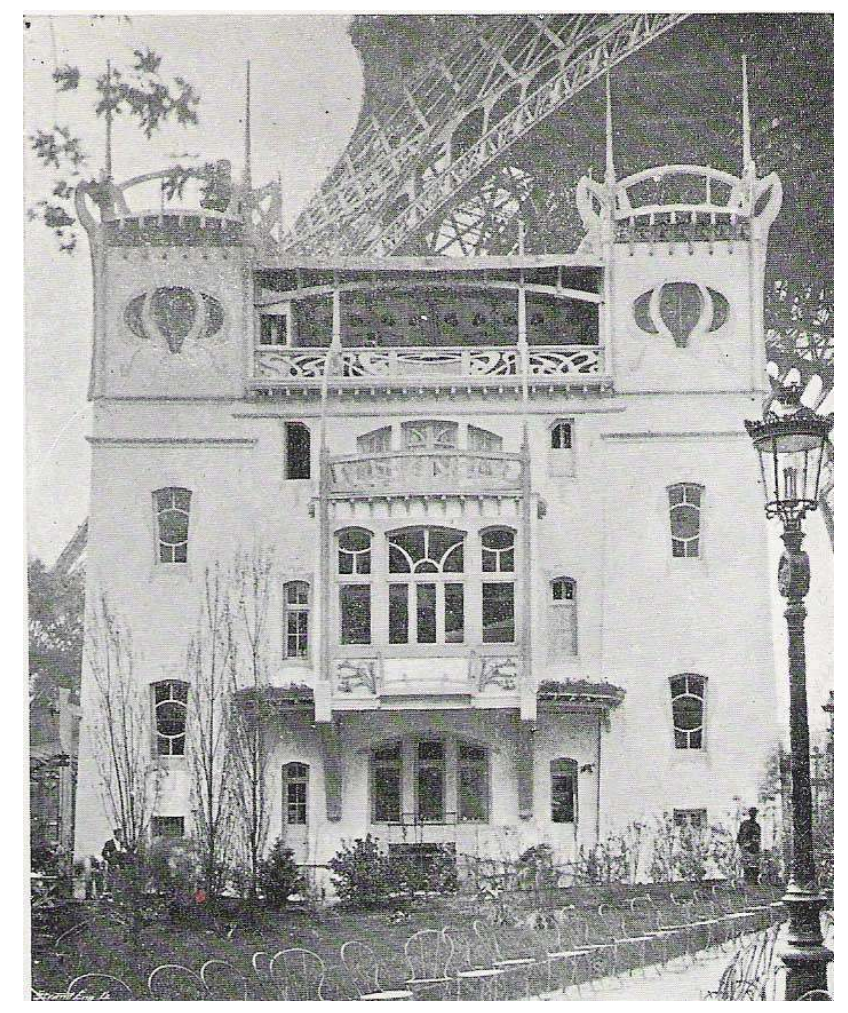

Dans A. Barthélémy, « L'Architecture nouvelle à l'Exposition », Art et décoration, juillet 1900, p. 14.

En lien, sans doute, avec sa nature éphémère, le Pavillon bleu est construit en bois. Le bois permet le développement de grandes arches qui mettent en avant la structure et concourent à un effet de légèreté. Pourtant, comme le note Étienne du Mesnil du Bigot, ces arches ont un rôle plus plastique que fonctionnel ; une structure plus modeste aurait suffi à soutenir le velum ${ }^{24}$. Un autre matériau très présent sur les façades est la faïence ${ }^{25}$ : une frise entre les premier et deuxième étages accueille des motifs végétaux linéaires et l'enseigne du restaurant. Sa place importante caractérise bien l'intérêt de l'architecture Art nouveau pour la variété des matériaux, intérêt particulièrement prononcé dans les créations de Serrurier, qui reprend l'usage de la faïence pour la villa «L'Aube » et le château de La Cheyrelle ${ }^{26}$.

Mais l'un des éléments les plus originaux du Pavillon bleu est assurément sa bichromie bleue et jaune. Si la couleur bleue trouve naturellement sa place dans un restaurant de ce nom, la présence d'une couleur jaune orangée est connue par le témoignage du critique A. Barthélémy ${ }^{27}$ et, surtout, par une précieuse lithographie d'Eugène Grasset représentant le restaurant en quatrième de couverture d'un menu ${ }^{28}$ (ill. 3). 
III. 3 : Menu du Pavillon bleu, Eugène Grasset

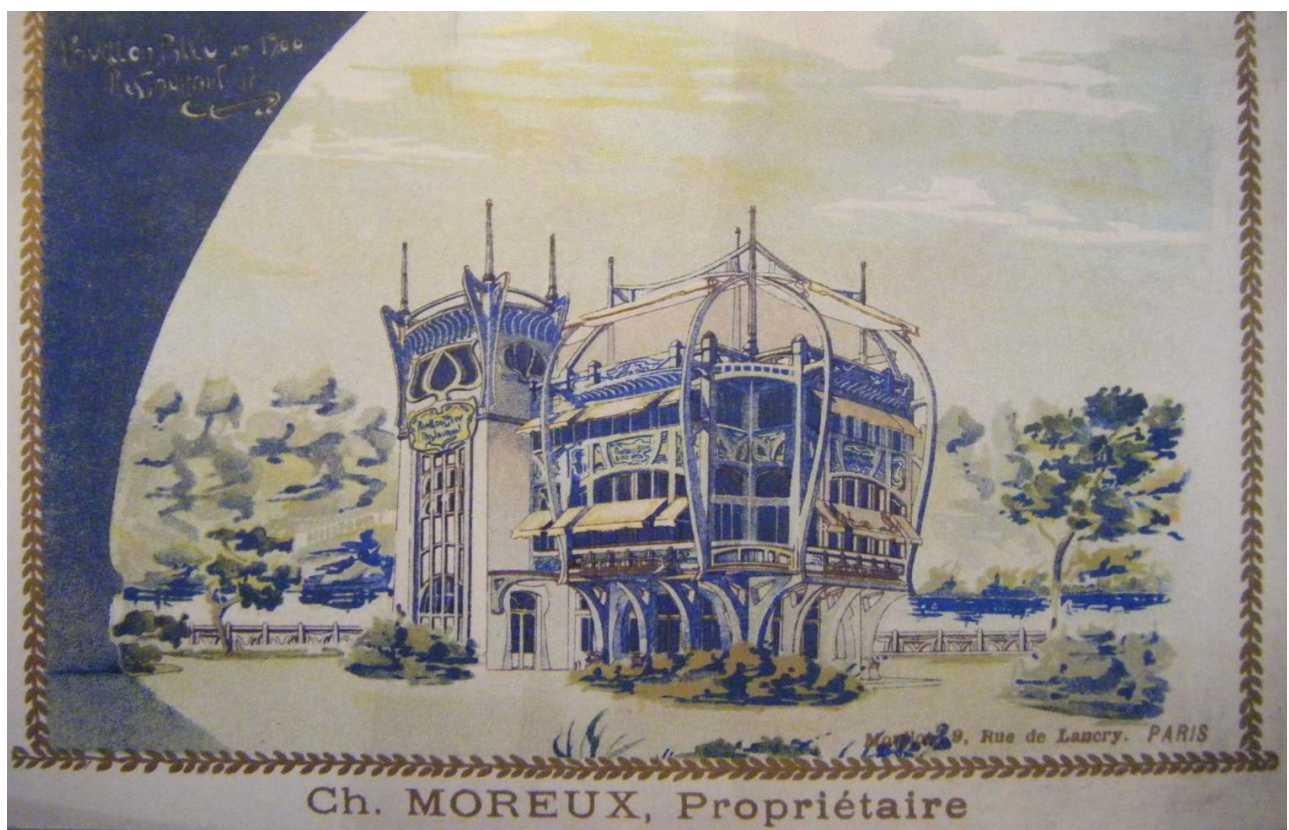

PAVILLON BLEU, 1900, À SAINT-CLOUd ET À L'EXPOSITION, 1900, 16 X 10 CM, LITHOgRAPHIE, ARCHIVES de PARIS, FONDS LEgRAND, D 29 z 54

CL. M.-A. THARAUD.

Il semble ainsi que le velum, les stores et le fond des faïences soient jaunes, la structure en bois et les motifs des faïences, bleus. Cette bichromie est reprise par Grasset dans la couverture du menu: une jeune femme, au visage botticellien typique de Grasset, boit une coupe de champagne, vêtue d'une robe jaune sur fond de paysage bleu (ill. 4). 
III. 4 : Menu du Pavillon bleu, Eugène Grasset

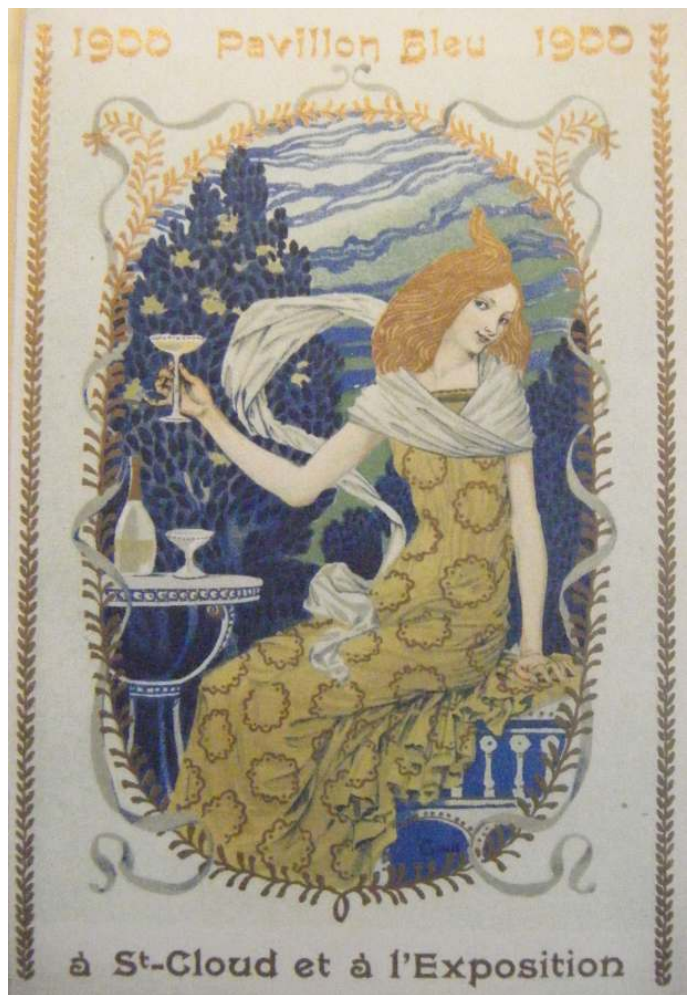

PAVILlon bleU EN 1900, RESTAURANT, CH. MOREUX PRopriÉtalRE, 1900, 16 X 10 CM, LITHOgRAPHIE, ARCHIVES DE PARIS, FONDS LEgRAND, D 29 Z 54

CL. M.-A. THARAUD.

15 S'il n'est guère possible de connaître l'intensité des couleurs, il est certain que la bichromie du restaurant constitue un élément à la fois rare et attrayant.

16 La décoration intérieure n'est révélée que par une photographie d'une salle de restaurant ${ }^{29}$, très probablement située au rez-de-chaussée étant donné les dimensions modestes de la pièce et l'absence des grandes baies des étages (ill. 5). 


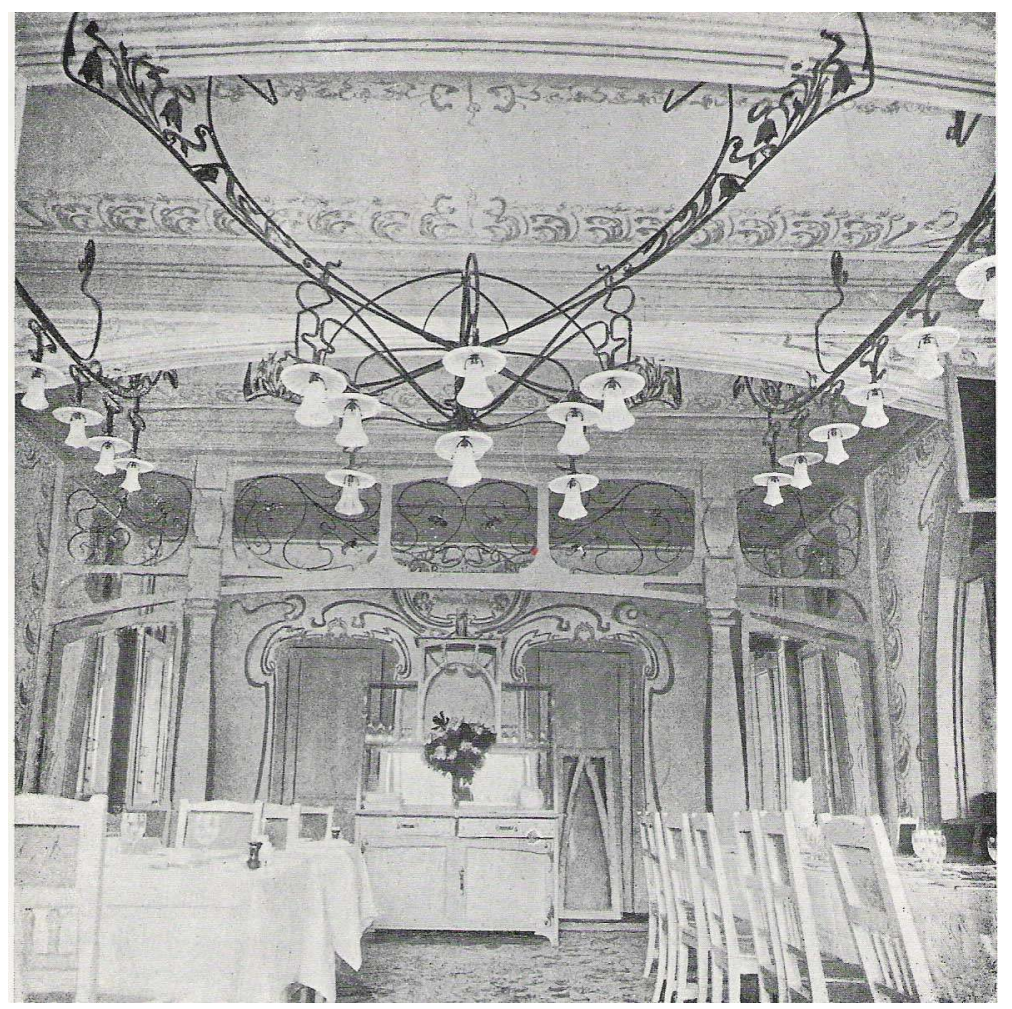

Dans A. Barthélémy, « L'Architecture nouvelle à l'Exposition », Art et décoration, juillet 1900, p. 15.

Sur les couleurs de la décoration et des meubles, on sait seulement que les salles sont « claires et confortables ${ }^{30}$ ». La photographie laisse penser que le bois du mobilier est très clair, blanc; mais Étienne du Mesnil du Buisson a remarqué qu'à l'époque, Serrurier préférait le bois $n u^{31}$. Au demeurant, l'intérieur offre un contraste saisissant entre une décoration exubérante et un mobilier orthogonal d'une grande sobriété. Les murs et les plafonds sont couverts de motifs linéaires abstraits, typiquement belges - dans la continuité de la cage d'escalier de l'hôtel Tassel de Horta -, et les lustres s'épanouissent en une ferronnerie surabondante, caractéristique de l'art de Serrurier. Il reprend en 1901 le motif du grand lustre dans la salle de billard du château de La Chapelle-en-Serval.

De fait, le Pavillon bleu constitue la manifestation d'un Art nouveau affirmé et triomphant, qui ne peut manquer de susciter des réactions partagées.

\section{Réception critique}

19 Événement international exceptionnel, l'Exposition universelle suscite de nombreux commentaires dans la presse de l'époque. Aussi l'Art nouveau, parce qu'il soulève des interrogations, bénéficie-t-il d'une riche réception critique. Si les critiques d'art s'accordent sur la nécessité d'un art nouveau, la forme qu'il doit revêtir est alors le sujet de vifs débats, accrus par l'Exposition. Les positions théoriques des critiques sont complexes, car l'Art nouveau est perçu comme un mouvement hétérogène et en évolution, dont il faut éliminer ou favoriser certains traits. Toutefois, c'est un Art nouveau rationnel et modéré, s'inspirant de la nature et se référant aux traditions stylistiques qui plaît majoritairement aux critiques français ${ }^{32}$. La réception critique du 
Pavillon bleu doit donc être appréhendée dans ce contexte de polémiques et de définitions. Elle n'est pas univoque, bien que le restaurant échappe au mépris que rencontrent certaines œuvres, allemandes notamment, et reçoive des commentaires souvent élogieux. L'article d'A. Barthélémy lui est hautement favorable. Il débute par des louanges : "Je féliciterai tout d'abord M. Dulong ", admire le "goût très original et très plaisant de la ligne rationnelle", loue le refus du "luxe habituellement clinquant des cabarets à la mode», et voit "la formule légitime, originale et réservée, du vrai Restaurant moderne $~_{33}$. De même, Roger Marx, séduit, ose une comparaison navale :

Le «Pavillon bleu» ne laisse pas de séduire par le chatoiement d'une couleur dominante, par le parti de la charpente visible; il est comme encerclé dans de fines poutrelles équarries, qui fusent et s'infléchissent en se raccordant aux traverses du faîte. Est-ce à cause du système surplombant - le pavillon est plus large à l'étage qu'à sa base - ou bien en raison des ouvertures pratiquées sans interruption presque, sur toutes les faces? Le "Pavillon bleu » fait songer à une construction flottante, à deux salles de paquebots superposées ${ }^{34}$.

Mais le Pavillon bleu est aussi identifié comme produit du "modern style», cet Art nouveau à la mode et populaire. Signe de la notoriété du restaurant, l'écrivain Jean Lorrain imagine cette remarque d'un personnage parcourant l'Exposition:

À propos de restaurant, avez-vous vu le "Pavillon-Bleu" de Saint-Cloud, [...] près de la tour Eiffel, vis-à-vis le "Palais lumineux", près du petit lac? C'est délicieux d'architecture, très modern style; mais ces poutrelles peintes en bleu sont d'un effet charmeur ! ${ }^{35}$

Or, le «modern style » auquel se rattachent les courbes et la décoration envahissante du Pavillon bleu, est souvent considéré par les critiques comme une version de l'Art nouveau excessive et condamnable. En conséquence, le Pavillon bleu suscite aussi des réticences. Le critique Charles Saunier, quoique plutôt partisan de l'Art nouveau, reste sur la défensive :

Le "Pavillon Bleu" est assurément modern style. Tout y est : bois courbé, volutes, couleurs voyantes. Nous n'osons cependant le louer, car la seule fantaisie a une trop large part dans tout cela. ${ }^{36}$

C'est donc cette "fantaisie», présente dans les arches élancées et la polychromie du Pavillon, et qui fait toute sa virtuosité, que blâme Saunier. En 1902, Rémy de Gourmont, commentant l'essai de Roger Marx consacré à l'Exposition universelle, se révèle encore plus méprisant en confrontant le restaurant à l'affirmation d'Ernest Renan, que «l'esprit de l'homme n'est jamais absurde à plaisir » :

Ne disons donc cela [...] ni de la Porte monumentale que nous traiterons alors de mystérieuse, ni du Pavillon bleu (encore qu'il est bien tentant de n'y voir qu'une baleine qui, ayant mis ses côtes par-dessus son lard, se dresserait sur les nageoires pour faire la belle) $[. . .]^{37}$

De la « construction flottante » évoquée par Marx, Gourmont a ainsi fait une baleine. Il ne faut point cependant s'étonner de cette comparaison dépréciative. Arsène Alexandre, dans un article du Figaro, recourt à des images beaucoup plus méprisantes pour évoquer le style «nouille ${ }^{38}$ " avec lequel le Pavillon bleu n'est pas sans lien. L'audace même de l'édifice est cause des reproches, finalement rares, qu'il suscite. 


\section{Bilan financier}

24

Au-delà d'un édifice emblématique de l'Art nouveau, le Pavillon bleu a été conçu dans un but publicitaire et mercantile orchestré par son concessionnaire, Charles Moreux. Or, il semble bien que l'entreprise ait été un échec financier. La presse s'avère peu renseignée sur ce sujet. Par exemple, elle relate bien un dégât des eaux, mais sans rendre compte de sa gravité ${ }^{39}$; les Archives nationales révèlent, elles, l'étendue du dommage et montrent que Moreux a demandé en vain une indemnitét ${ }^{40}$. De même, il ne faut pas se fier aux spéculations des journalistes :

Dans un but intéressé, on a fait courir le bruit que la plupart des restaurants de l'Exposition ne faisaient pas leurs affaires, et que la situation des plus importants était compromise. Il n'est pas inutile de démentir ces assertions, notamment en ce qui concerne le Pavillon Bleu. La situation prospère de cet établissement est, d'ailleurs, connue de tous ${ }^{41}$.

Le dossier conservé aux Archives nationales contenant la correspondance entre Moreux et l'administration de l'Exposition, puis le procès qu'il lui intente, constitue une source bien plus fiable et révélatrice des difficultés financières, réelles, de Moreux. D'une part, il demande une diminution de sa redevance, celle-ci étant fort élevée (79 700 francs) ; sa demande est rejetée ${ }^{42}$. D'autre part, il attaque l'État en justice après s'être vu refuser un dédommagement qu'il justifie ainsi :

Étant sur le point de perdre trois cent mille francs dans cette affaire, résultat de toute une vie de travail et d'économies, et ayant une nombreuse famille tant qu'en enfants, qu'en vieux parents à soutenir, je ne pouvais ne pas relever les torts qui me sont causés par votre Administration, [...] retards de l'Exposition et aussi le nombre considérable de restaurants en plus de ceux qu'on avait mis en adjudication et qui étaient seuls indiqués sur le plan qui nous a été vendu ${ }^{43}$.

Ces arguments sont développés dans un mémoire adressé par l'avocat de Moreux au contentieux du Conseil d'État, repris dans la sentence du jury arbitral du contentieux du 30 mai 1903, et enfin dans la décision finale du Conseil d'État du 13 mai 1904. Moreux estime avoir perdu « une somme de 189257,32 francs $^{44}$ », et demande 234781 francs de dommages à l'état, qu'il estime responsable de deux torts principaux: avoir permis l'installation d'autres restaurants après l'adjudication du sien, ce qui l'a «induit [...] en erreur sur l'étendue des avantages que devait comporter la concession », et avoir pris du retard dans les travaux du Champ-de-Mars, laissé « jusqu'au 5 juin 1900, [...] dans l'état d'un simple chantier ", " sans éclairage le soir ", avec à proximité du Pavillon bleu " une voie ferrée et une grue ", éléments nuisibles à la fréquentation du restaurant. Mais Moreux est débouté car, entre autres, «l'administration se réserve le droit de concéder autant d'établissements similaires ou non », le Champ-de-Mars « est en état de viabilité dès l'ouverture de l'Exposition » et « l'État n'a pris aucun engagement relatif à l'éclairage de l'Exposition le soir ${ }^{45} »$. Il est de fait difficile de déterminer si les déboires de Moreux proviennent bien de défaillances de l'administration ou s'ils lui sont imputables, comme l'estime le Conseil d'État qui conclut que «les mécomptes dont se plaint M. Moreux doivent être plus vraisemblablement attribués au luxe de ses installations et au prix élevé de ses repas $»^{46}$.

Le Pavillon bleu n'a donc certainement pas été une bonne affaire financière pour Moreux. Il a dû néanmoins lui procurer une importante et brillante publicité pour le restaurant de Saint-Cloud.

Livraisons de l'histoire de l'architecture, 19 | 2010 
ignore combien de temps le Pavillon bleu a survécu à l'Exposition. D’après Étienne du Mesnil du Bigot, un film de la Cinémathèque française le montre debout après le démontage de l'Exposition ${ }^{47}$, mais aucun document des Archives nationales n'atteste sa présence après 1900. De fait, en dépit d'une large visibilité au sein de l'Exposition universelle et d'une bonne réception critique, son caractère éphémère a fait méconnaître cet édifice dont une part de l'audace résidait dans une polychromie qui ne peut être aujourd'hui rendue. D'une élégance flamboyante, le Pavillon bleu marque le succès de l'association entre un talentueux artiste décorateur engagé dans l'Art nouveau et un architecte sûrement moins brillant mais compétent, et fait le pont entre Art nouveau français et Art nouveau belge. Ainsi, alors que la première vocation du Pavillon bleu était d'ordre lucratif, sa véritable réussite est incontestablement artistique.

\section{NOTES}

1. Françoise Le Bigot du Mesnil du Buisson, Étienne du Mesnil du Buisson, Serrurier-Bovy un créateur précurseur 1858-1910, Dijon, Faton, 2008, 300 p., 299 p.; Françoise Bigot du Mesnil du Buisson, Gustave Serrurier, 1858-1910 (Serrurier-Bovy) parcours d'un architecte à l'aube du XX siècle, Lille, ANRT, 2006, 3 vol., Étienne du Mesnil du Buisson, L'Cuvre attestée de Gustave Serrurier (1858-1910), Lille, ANRT, 2007, 5 vol.

2. Exposition universelle de 1900 : les plaisirs et les curiosités de l'Exposition, Paris, Chaix, 310 p., p. 138.

3. . Photographie sur la base Images-Mémoire du site: http://www.culture.gouv.fr/culture/ inventaire/patrimoine.

4. Alfred Picard, Exposition universelle internationale de 1900 à Paris. Rapport général administratif et technique, Paris, Imprimerie nationale,

1902

1902-1903, t.7, 414 p., p. 191.

5. Exposition universelle de 1900 : les plaisirs..., op. cit., p. 138. Cf. aussi Henry Lapauze, Max de Nansouty, et al., Le Guide de l'Exposition de 1900, Paris, E. Flammarion, [1900], 540 p., p. 32, ou 1900. Paris Exposition, guide pratique du visiteur de Paris et de l'Exposition..., Paris, Hachette, 1900, 484 p., p. 172 : « Restaurant du Pavillon bleu, près de la Tour Eiffel. Établissement de luxe. Carte chiffrée. » 6. Premier menu: «Dîner du 25 octobre 1900/Crème de volaille/Huîtres d'Ostende/Saumon sauce mousseline/Filet de bœuf Périgueux/Poularde flanquée de perdreaux/Salade de chicorée/ Bombe glacée/Gaufrettes/Corbeille de fruits", "Vins/Barsac/ Médoc en carafe/Château Margaux 1892/Champagne Saint Marceaux/Café-liqueurs ». Deuxième menu : «Déjeuner du 5 novembre 1900/Hors d'œuvre variés/Marenne extra/Langouste américaine/Tournedos grillés Béarnaise/Pommes soufflées/Caneton à la rouennaise/Salade de légumes/Soufflé chocolat/ Corbeille de fruits", "Vins/Mâcon/Graves en carafe/Pommard Champagne frappé/Caféliqueurs ", Archives de Paris, fonds Legrand, D 29 Z 56.

7. Alfred Picard, Exposition..., op. cit., t. 7, p. 195.

8. Arch. nat., $\mathrm{F}^{12} 4369$, restaurant Moreux - convention.

9. Ibid., décision du Conseil d'État, 13 mai 1904, p. 2.

10. Il écrit pour la revue Art et décoration dirigée par Gustave Soulier, un admirateur de Serrurier sûrement bien renseigné ; Étienne du Mesnil du Buisson, L'Euvre..., op. cit., p. 300. Soulier 
consacre un article élogieux au magasin de Serrurier et Dulong: Gustave Soulier, «L'Art dans l'Habitation ", Art et décoration, avr. 1900, p. 105-117.

11. A. Barthélémy, «L'Architecture nouvelle à l'Exposition », Art et décoration, juil. 1900, p. 14.

12. Alfred Picard décrit attentivement le Pavillon bleu sans citer Serrurier: Picard, Rapport général..., t. 7, p.247-248.

13. Roger Marx, La Décoration et les industries d'art à l'Exposition universelle de 1900, Paris, Ch. Delagrave, 1901, 133 p., p. 26.

14. Sur Dulong : Françoise Bigot du Mesnil du Buisson, Gustave Serrurier..., op. cit., p. 364-374.

15. Arch. nat., $\mathrm{F}^{12} 4445$.

16. Alfred Picard, Exposition.., op. cit., t. 7, p. 230.

17. La belle-famille de Dulong a conservé ses archives à La Cheyrelle (Cantal), jusqu'à leur dispersion en 1990. Françoise Bigot du Mesnil du Buisson, Gustave Serrurier..., op. cit., p. 364.

18. Ibid., p. 371.

19. Sur Serrurier : Françoise Bigot du Mesnil du Buisson, Étienne du Mesnil du Buisson, SerrurierBovy..., op.

cit.

20. Nos recherches aux Arch. nat. et aux Archives de Paris ont été vaines.

21. Les témoignages sont parfois erronés: A. Picard, par ailleurs assez précis, ne mentionne qu'une tour d'angle et qu'un étage au lieu de deux ; A. Picard, Exposition..., op. cit., t.7, p. 247-248. Pour l'intérieur, les rares témoignages ne peuvent être vérifiés.

22. Photographie dans A. Barthélémy, «L'Architecture... », op. cit., p. 14.

23. Picard n'évoque qu'« une tour d'angle abritant l'escalier principal», mais l'autre tour devait abriter un escalier de service. A. Picard, Exposition..., op. cit., p. 247.

24. Étienne du Mesnil du Buisson, L'Euvre..., op. cit., p. 300.

25. Alfred Picard, Exposition..., op. cit., t.7, p. 248.

26. Étienne du Mesnil du Buisson, L'CEuvre..., op. cit., p. 300.

27. «Un ensemble de bon goût, d'harmonie discrète et sûre, où les bleus et les jaunes jouent admirablement », A. Barthélémy, «L'Architecture... », op. cit., p. 16.

28. Archives de Paris, fonds Legrand, D 29 Z 56.

29. A. Barthélémy, "L'Architecture... », op. cit., p. 15.

30. Ibid., p. 16.

31. Étienne du Mesnil du Buisson, L'Eeuvre..., op. cit., p. 301.

32. Sur la réception critique de l'Art nouveau à l'Exposition universelle : Marie-Amélie Tharaud, L'Art nouveau à l'Exposition universelle de 1900, thèse pour le dipl. d'archiviste paléographe, dactyl., 2009, 2 vol., p. 197-338.

33. A. Barthélémy, «L'Architecture... », op. cit., $1^{\text {re }}$ citation p. $13,2^{\mathrm{e}}$ citation p. $14,3^{\mathrm{e}}$ et $4^{\mathrm{e}}$ citations p. 16.

34. Roger Marx, La Décoration..., op. cit., p. 26.

35. Jean Lorrain, Philippe Martin-Lau éd., Mes Expositions universelles (1889-1900), Paris, Honoré Champion, 2002, 434 p., p. 208.

36. Charles Saunier, «Les Petites constructions de l'Exposition ", L'Art décoratif, nov. 1900, p. 58.

37. Rémy de Gourmont, «Sur l'Art nouveau », Le Mercure de France, mars 1902, p. 700.

38. Arsène Alexandre, « Modern style ", Le Figaro, $1^{\mathrm{er}}$ sept. 1900, p. 1.

39. "Par suite de la rupture d'un barrage d'égout, les caves du "Pavillon bleu" ont été inondées ce matin. Les pompiers, immédiatement requis, ont travaillé à l'épuisement de l'eau toute la matinée. À midi, les caves étaient remises en l'état. ", "À l'Exposition », Le Temps, 25 juin 1900, p. 
40. Arch. nat., $\mathrm{F}^{12} 4369$ : lettres de Moreux, 25 juin et 25 juil. 1900 ; lettre du service de la voirie, 11 août 1900 : l'indemnité est refusée car « les eaux de l'égout se sont répandues dans les caves du restaurant par suite du mauvais état de la construction ».

41. Jean de La Tour, «À travers l'Exposition », Le Figaro, 10 juil. 1900, p. 3.

42. Arch. nat., $\mathrm{F}^{12} 4369$, lettre du commissaire général à Moreux, 26 oct. 1900.

43. Arch. nat., $\mathrm{F}^{12}$ 4369, lettre de Moreux au commissaire général, 11 sept. 1900.

44. Arch. nat., $\mathrm{F}^{12}$ 4369, sentence du jury arbitral, Conseil d'État, contentieux, 30 mai 1903.

45. Pour toutes les citations, Arch. nat., $\mathrm{F}^{12}$ 4369, décision du 13 mai 1904, Conseil d'État, contentieux.

46. Arch. nat., $\mathrm{F}^{12}$ 4369, sentence du jury arbitral, Conseil d'État, contentieux, 30 mai 1903.

47. Étienne du Mesnil du Buisson, L'EEuvre..., op. cit., p. 294. Nous n'avons pu vérifier.

\section{RÉSUMÉS}

Restaurant de luxe édifié pour l'Exposition universelle de 1900, le Pavillon bleu est la première œuvre conjointe du célèbre décorateur belge Gustave Serrurier-Bovy et de l'architecte français René Dulong, dont l'association allait durer sept ans. Il constitue une enseigne publicitaire pour un établissement du même nom établi à Saint-Cloud. Idéalement situé sous la tour Eiffel afin d'attirer la clientèle, il se distingue de l'éclectisme dominant pour adopter un style Art nouveau séduisant et audacieux, dont l'originalité provient d'une bichromie bleue et jaune appliquée à une structure en bois singulièrement mise en valeur. La collaboration entre Serrurier, décorateur, et Dulong, architecte, a ainsi donné lieu à une œuvre à la fois harmonieuse et affirmée, relativement bien accueillie par la critique dans un contexte pourtant hostile à toute manifestation ostentatoire de modernité. Le Pavillon bleu échoue cependant dans sa vocation première, mercantile, comme en témoignent les déboires financiers de son concessionnaire, qu'il faut peut-être attribuer aux défaillances du commissariat de l'Exposition universelle ; sa réussite est donc véritablement d'ordre artistique. Méconnu de l'historiographie du fait de son caractère éphémère - il a disparu après la fermeture de l'Exposition à une date inconnue -, il n'en constitue pas moins un édifice brillant et emblématique d'un Art nouveau de style franco-belge.

A luxury restaurant built for the Universal Exposition of 1900, the Pavillon bleu is the first joint work of the famous Belgian decorator Gustave Serrurier-Bovy and the French architect René Dulong, whose association was to last seven years. The Pavillon bleu is an advertising sign for an institution of the same name established at Saint-Cloud. Ideally located under the Eiffel Tower to attract customers, it differs from dominant eclecticism to adopt an attractive and daring Art Nouveau style, whose originality comes from a blue and yellow bichromy applied to a singularly highlighted wooden structure. The collaboration between Serrurier, decorator, and Dulong, architect, has resulted in a work at once harmonious and confident, relatively well received by critics in a context yet hostile to any ostentatious manifestation of modernity. The Pavillon bleu, however, fails in its primary, mercantile, purpose, as show the owner's financial woes, which may be put down to failures from the Universal Exposition's commission; thus, its success is truly an artistic one. Ignored from historiography because of its ephemeral nature - the Pavillon bleu 
disappeared after the closure of the exhibition at an unknown date - it is nonetheless a brilliant and emblematic building of a Franco-Belgian Art nouveau style.

Das aus Anlass der Weltausstellung 1900 gebaute Luxusrestaurant Pavillon bleu ist das erste gemeinsame Werk des Dekorateurs Gustave Serrurier-Bovy und des französischen Architekten René Dulong, die anschließend sieben Jahre lang zusammenarbeiten sollten. Das unter dem Eiffelturm errichtete Restaurant, das als Werbemittel für eine gleichnamige in Saint-Cloud gelegene Anstalt dienen sollte, war durch diese ideale Lage besonders attraktiv für die Gäste. Das Gebäude unterschied sich von überall herrschendem Eklektizismus durch elegante und gewagte Jugendstilformen. Besonders kreativ war die Zweifarbigkeit des Gebäudes, dessen Holzgerüst so durch blaue und gelbe Farben unterstrichen wurde. Durch die enge Zusammenarbeit des Dekorateurs Serrurier mit dem Architekten Dulong entstand also ein harmonisches eben sowie aussagekräftiges Werk. Es wurde von der Kritik relativ gut aufgenommen, obwohl in der damaligen Umgebung demonstrative Ausdrücke der Moderne negativ angesehen waren. Was die ursprünglichen kommerziellen Absichten des Pavillon bleu betrifft, wurde es ein totales Scheitern, wie es die finanziellen Unannehmlichkeiten des Lizenzinhabers bezeugen, die vielleicht eher den Schwächen des Kommissariats der Weltausstellung zugeschrieben werden konnten. Der Pavillon bleu wurde also vor allem ein künstlerischer Erfolg. Dieses vergängliche Gebäude, das irgendwann nach dem Schluss der Weltausstellung verschwand, wurde natürlich von den Kunsthistorikern verkannt. Aber dieses prächtige Gebäude erweist sich doch als ein besonders repräsentatives Beispiel für den belgisch-französischen Jugendstil.

\section{AUTEUR}

\section{MARIE-AMÉLIE THARAUD}

Marie-Amélie Tharaud, née en 1985, est conservateur du patrimoine actuellement en formation à l'Institut national du patrimoine. Archiviste paléographe, elle a soutenu une thèse sur L'Art nouveau à l'Exposition universelle de 1900 à l'École nationale des chartes en 2009, sous la direction de Jean-Michel Leniaud. Elle poursuit ses recherches en doctorat à l'École Pratique des Hautes Études. Elle a également collaboré à l'ouvrage L'Art nouveau de Jean-Michel Leniaud, Paris, Citadelles \& Mazenod, 2009, 620 p., pour les parties sur la peinture. Adresse électronique : tharaud@hotmail.com 